Glazov EA et al. Supplemental Figure S3

Fig S3a.

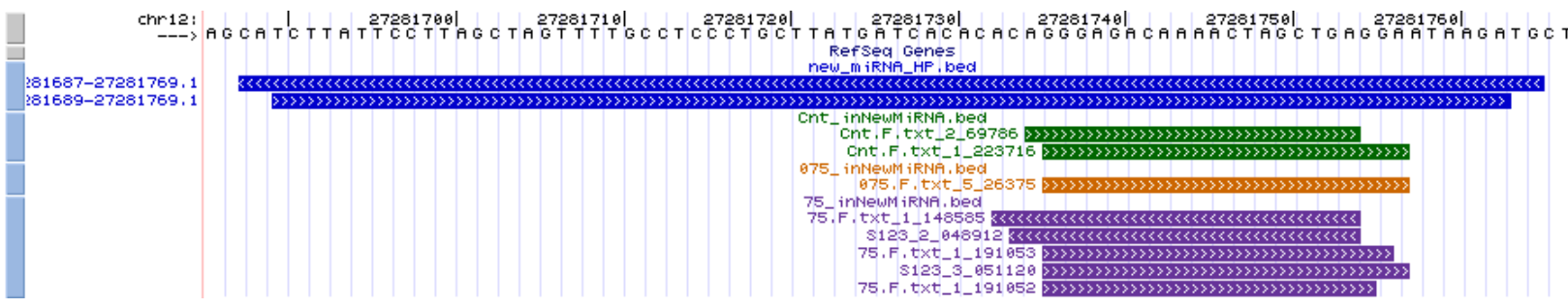

Fig S3b.

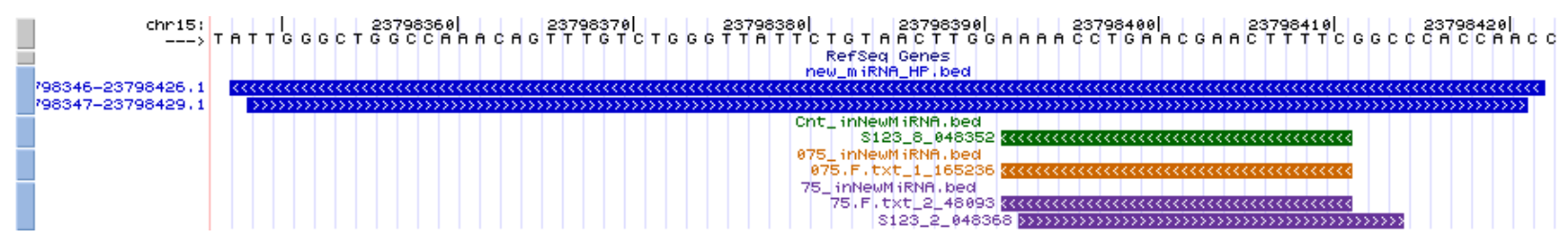

Fig S3c.

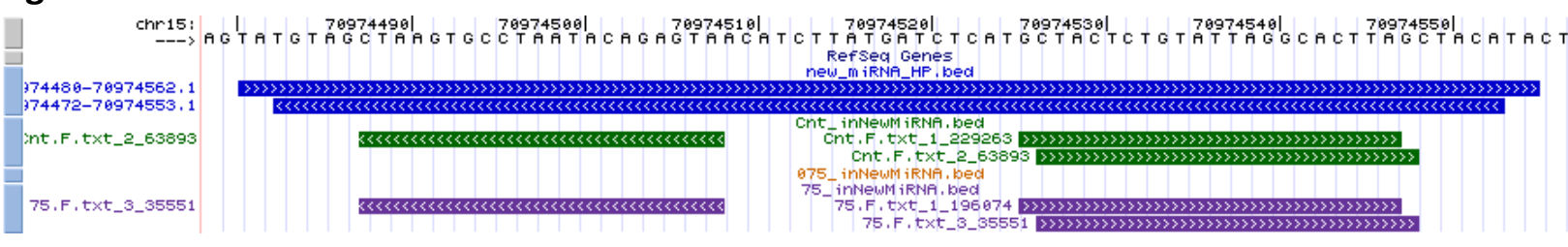

Fig S3d.

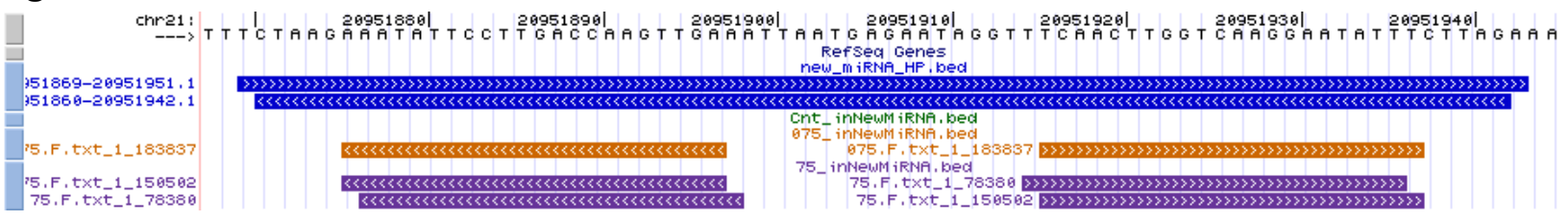

\title{
Fig S3e.
}

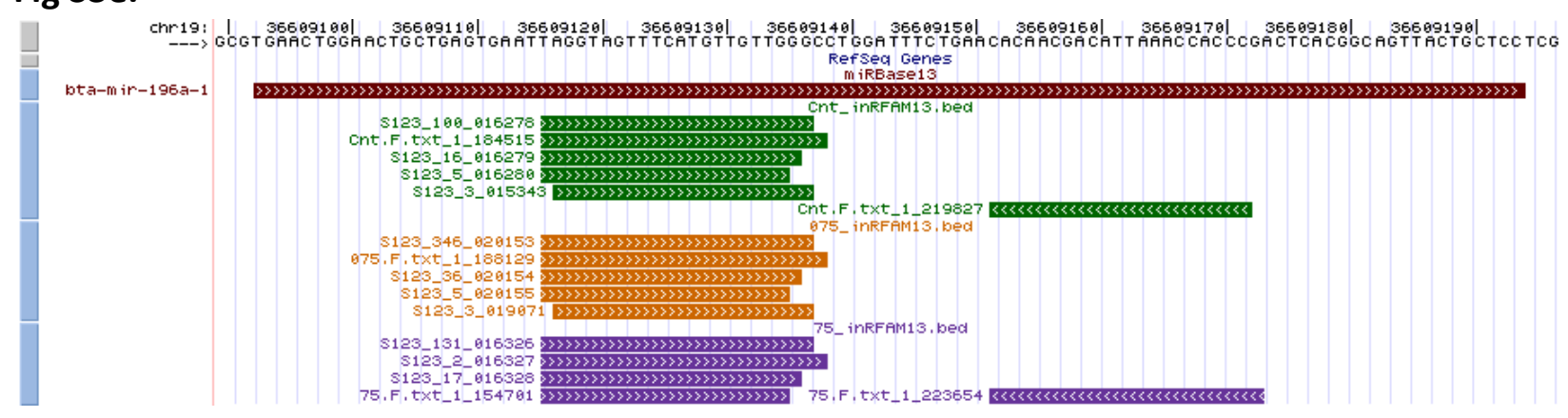


Glazov EA et al. Supplemental Figure S3

Fig S3f.

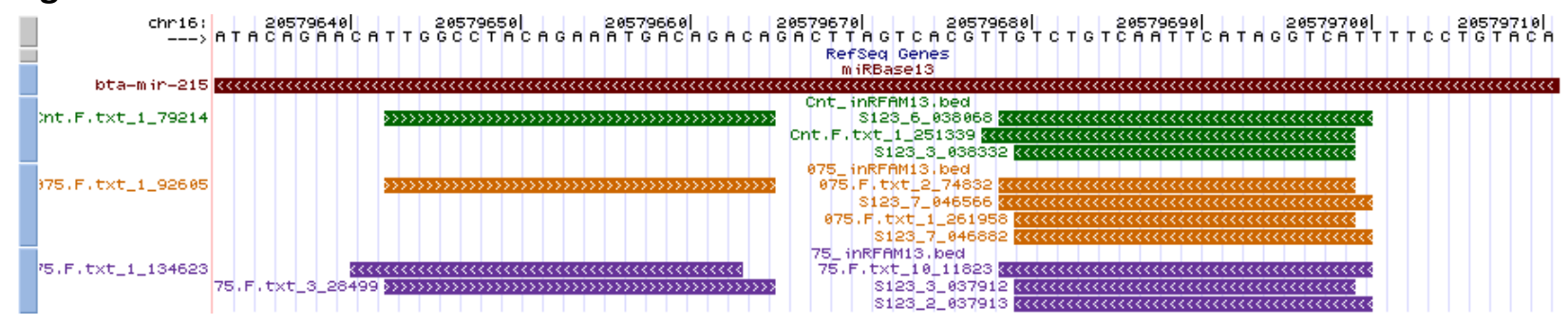

Fig S3g.

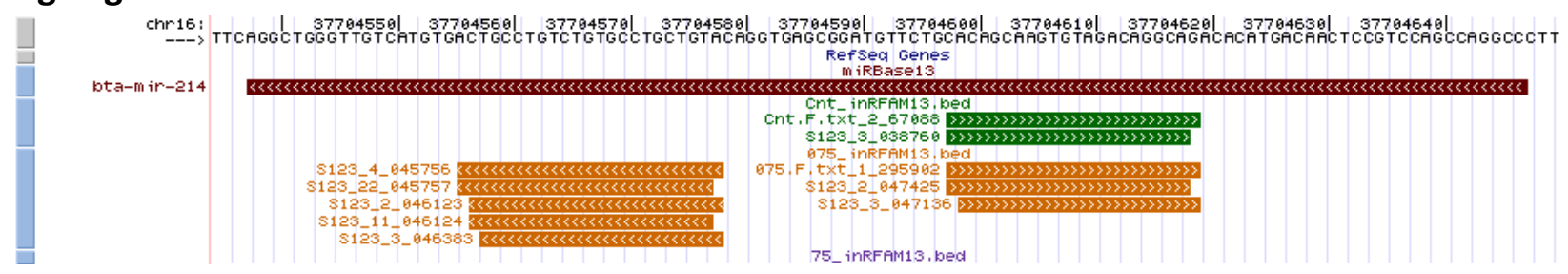

Fig S3h.

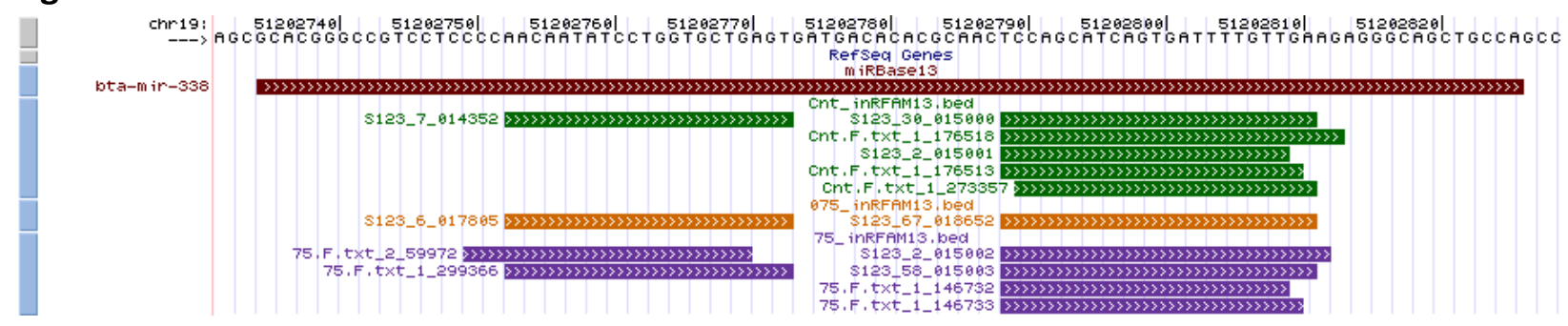

Supplemental Figures S3a-S3h. Bidirectional miRNA candidates. (S3a-S3d) New miRNA candidates that have evidence of transcription and predicted hairpin-like structures on both genomic strands. (S3e-S3g) Known miRNA candidates that have evidence of transcription and predicted hairpin-like structures on both genomic strands. (S3h) Cow miRNA bta-mir-338 that has evidence of bidirectional transcription in other species (Tyler et al. 2008). The figures shows UCSC genome browser screens displaying relative positions of predicted miRNA hairpin-like precursors (blue) or known miRNA precursor (deep red), and sequence tags originating from this loci in three small RNA libraries: mock-infected control - green, $\mathrm{MOI}$ 0.75 library - dark orange, MOI 7.5 library - magenta. Arrowheads indicate alignment of sequences relative to the genomic strands. 